\title{
Constructing Digital Library Information Platform Based On Cloud Computing
}

\author{
Yubin Wang ${ }^{1}$, Jingyi bo ${ }^{1}$ and Weili $\mathrm{Xu}^{2}$ \\ ${ }^{1}$ College of Math and Information Science \& Technology, Hebei Normal University of \\ Science\& Technology \\ Qinhuangdao, Hebei, 066004, China, \\ ${ }^{2}$ The College of Business Administration, Hebei Normal University of Science \& \\ Technology \\ Qinhuangdao, Hebei, 066004, China, \\ $\left\{{ }^{1}\right.$ qhdwyb, ${ }^{2}$ qhdbjy\}@126.com
}

\begin{abstract}
With the development of cloud computing, more and more fields are leveraging it for application revolution. As the information resources of university libraries are becoming digital and networked, the portal of university libraries is now the important source of information and services. However, the access of information and services becomes harder because of the massive data resources and services. Besides, the cost on collection expenditure, network devices and resources integration is also increasing. To this end, we propose to construct a digital library information platform using cloud computing technique. Specifically, in his paper, we propose a reference architecture of a cloud based digital library information platform, and then focus on the resource allocation component. In our experiment, we evaluate the efficiency of our resource allocation algorithm.
\end{abstract}

Keywords: Digital library, Cloud computing, Resource allocation

\section{Introduction}

As the information resources of university libraries are becoming digital and networked, the portal of university libraries is now the important source of information and services. However, when providing massive data resources to all teachers and students, issues increasingly stand out. For example, the access of information and services is based upon Web servers, which become bottlenecks since the more and more data resources and services are required. Besides, if the client is directly connected to Web servers, it is likely to expose background data to intruders.

Recent years, cloud computing [1-2] provides a new solution for flexible computing capacity, efficient massive data analytics and inherent security mechanism. When cloud computing brings great revolution to IT field [3-4], it occurs to us that what it can do to libraries. With the development of Internet and Web 2.0, knowledge and information tend to grow infinitely, and the requirement of information resources from readers has gradually increased as well. Accordingly, the digital resources have to be expanded for the university libraries. However, it raises a big pressure in terms of collection expenditure, network devices and resources integration. In this situation, libraries cannot rely on improved hardware and increased funding to solve the problems technically and financially.

The solution is to leverage cloud computing for constructing a digital library platform. In this paper, we propose a reference architecture on constructing a cloud based digital library information platform. Specifically, we design four layers, i.e., infrastructure layer, platform layer, application layer and access interface layer. More specifically, we focus on one of the most important aspect, dynamic resource allocation, and present an efficient algorithm.

The remainder of this paper is organized as follows. Section 2 provides some background, 
and Section 3 reviews related work. The reference framework is discussed in Section 4. Then the resource allocation is introduced in Section 5. Empirical experiments of evaluating the allocation algorithm in Section 5 are conducted in Section 6. Finally, the paper is concluded in Section 7.

\section{Background}

\subsection{Cloud Computing}

As defined in [5], cloud computing is a variety of computing concepts that involve a large number of computers connected through a real-time communication network such as the Internet. Cloud computing is typically used to refer to network-based services, where the "cloud" is essentially Internet. Cloud computing relies on sharing of resources to achieve coherence and economies of scale, and aims to maximize the effectiveness of the shared resources [6].

From the definition of NIST [6], cloud computing has the following five characteristics: (1) Storage and computation of huge amount of data, which is the core of cloud computing. (2) Cost savings by providing variety of resources on demand with dynamic flexibility using pay-as-you-go pricing strategy; (3) Resource sharing by providing information infrustracture and abstracting resources such as physical servers, virtual machines, event and file processing capacity or task processes, and storage resources, which are served for various kinds of applications; (4) Rapid elasticity, which means that the system can be easily expanded in scale; and (5) Dynamic allocation, which achieves to automatically assign, real-time monitor and schedule resources.

Typical service models of cloud computing are categorized into three types: Software as a Service (SaaS) [7], Platform as a Service (PaaS) [8] and Infrastructure as a Service (IaaS) [9]. (1) SaaS is an on-demand software and usually are priced using a pay-as-you-go model. SaaS is typically accessed by users via a web browser. It has been widely applied in fields such as database management, virtualization, Customer Relationship Management (CRM), Management Information Systems, and Enterprise Resource Planning (ERP), etc. (2) In PaaS models; cloud providers deliver a storage or computing platform. PaaS offerings facilitate the deployment of applications without the cost and complexity of buying and managing the underlying hardware and software and provisioning hosting capabilities. (3) IaaS offers offer physical or virtual machines and other resources to cloud users. Then all details related to the infrastructure are handled by the cloud provider offerings.

\subsection{Applying Cloud to Libraries}

Griffey J first propose the concept of Cloud Librarians in 1008, and pointed out that it is important to leverage new techniques for serving customers when the technology is mature [10]. In the network era, as one of the most technically closely associated with IT field, libraries always keep trying to applying new techniques for application. With cloud computing, libraries can further improve the resources construction and service positioning based on cloud services.

Using the super power of data processing of cloud computing, jobs are automatically distributed over thousands of computers as smaller granularity tasks. Then, massive business data is rapidly analyzed, processed, stored and mined for valuable information, which can further be used for library services.

Applying cloud computing to libraries can help to integrate information resources. Integration is one of the most important concept in cloud computing, including hardwares, computing and storing capacity. With cloud based services, users can access information and services anywhere using any devices, without caring about the complicated background operation. The focus would be the information acquisition and sharing. In this way, library users can access existing resources anytime and real time.

Another benefit of cloud computing is saving costs. Traditionally, in order to ensure the 
normal operation of digital library, computers and servers have to be maintained, upgraded and updated. But in cloud computing environment, the routine maintenance procedure is taken by the cloud providers and problems with regards to hardware are no longer a concern.

In addition, the storage capacity of cloud computing enables libraries holds increasing information and resources. Moreover, cloud computing also provides supports for complicated computing procedures along with specific data structures. For example, indexing based on cloud is more effective compared to the traditional way. When users submit a retrieval request, the cloud allocates powerful computing capacity according to the request, complicated computing is executing on servers, and proper results are returned to users in satisfactory response rate.

\section{Related Work}

Koha [11], as a first open-sourced library automation system, was built on LibLime and distributed in a SaaS [12] way. The target users of Koha are small-sized public libraries, schools and professional libraries.

In 2006, Amazon released Elastic Compute Cloud (EC2) [13]. EC2 provides IT infrastructure services to different organizations, including storage and computing resources. The District of Columbia Public Library is using EC2 service to host their website [14], and Amazon Simple Storage Service (S3) [15] for backup. Besides, OhioLINK also uses Amazon Web Services (AWS) to host partial of their digital public resources [16].

In 2008, Google AppEngine [17] allows to construct Web applications using Google infrastructure. Eastern Kentucky University Library has moved some of their internal functions to Google tools [18]. For example, they use Google Docs to gather replies of website forms, and Google Calender for scheduling training and meeting, Google Analytics to collect data from websites, library catalogue and blogs.

In 2009, OCLC introduces the cloud-based services into libraries [19]. OCLC provides cloud-based, Web scale collaborative library management services to its members. Later, OCLC announced the pilot project of a new service, WorldCat Local, which will is provided across the Internet to eliminate costs to the library for hosting, operating and maintaining softwares [20].

\section{Framework}

In this section, we present a reference framework of cloud based digital library platform. As shown in Figure 1, we have four layers: access interface layer, application layer, platform layer and infrastructure layer. Figure 2 shows the deployment of our platform.

\subsection{Infrastructure Layer}

In order to integrate all the resources of libraries, all hardwares are connected through the cloud infrastructure. For higher level of resources management logic, virtualization technique is used to ignore the differences between hardware products, and also provides a unified description for each resource.

Virtualization breaks the barriers between different structures of physical devices. All hardwares are abstracted as a resource pool, and then dynamic allocation and workload balancing can be achieved. Besides, using virtualization for data replication and backup facilitates the process of zero down time migration and rapid disaster recovery, and therefore data security is ensured.

Virtualization allows integrating all the existing hardware resources as a huge resource pool, and resources are allocation according to the application requirement. As illustrated in Figure 2, virtualization can take one single server as a clustering of multiple virtual servers, and on the other hand, it can take multiple servers as a single virtual server as well. 


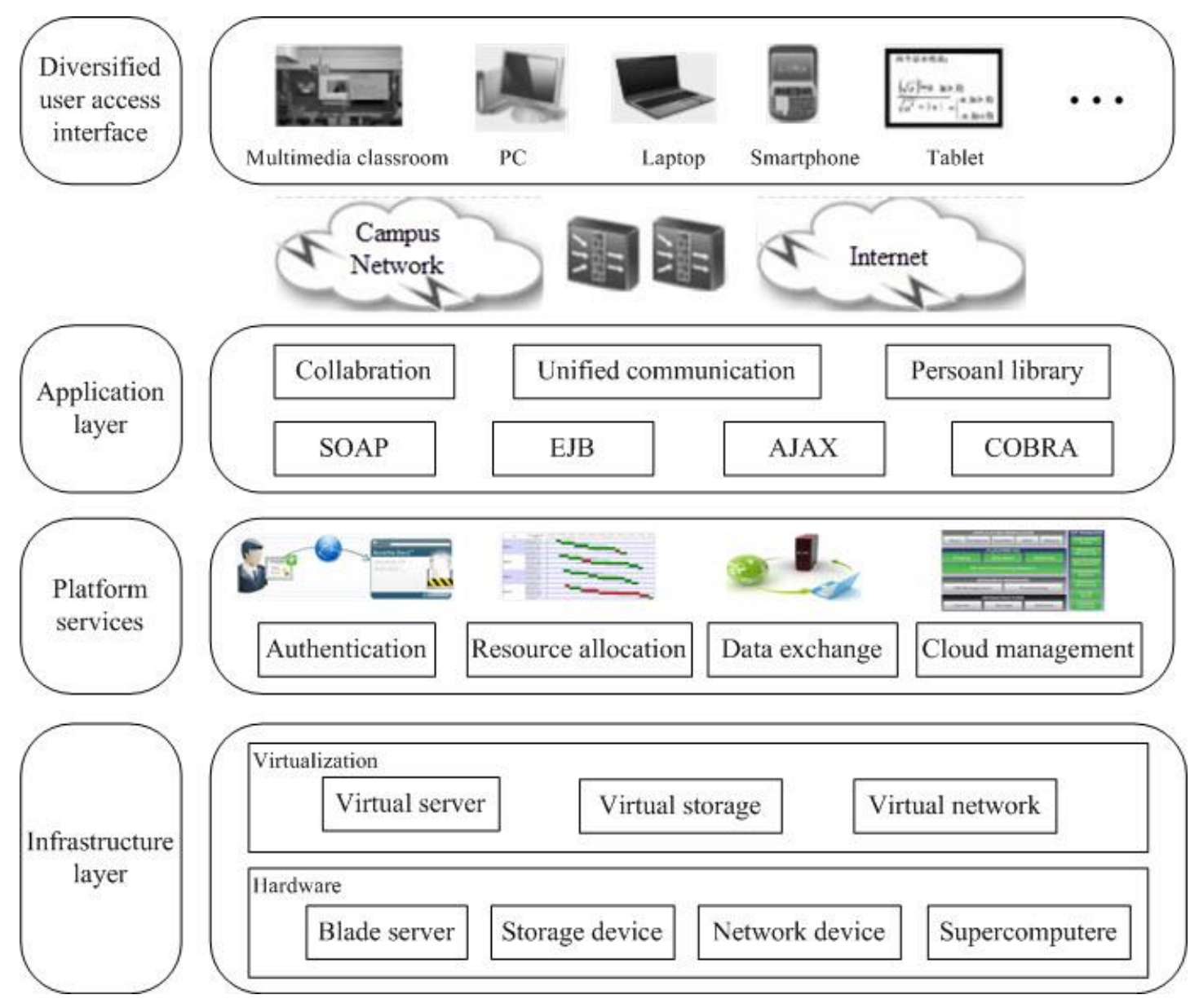

Figure 1. Framework of Cloud Based Digital Library Architecture

\subsection{Platform Layer}

This layer is built upon infrastructure layer, which is typically provided by the cloud vendors. However, this layer provides higher level services such as authentication, resource allocation, and data exchange and cloud management.

Besides, developers can also create more applications using the public environment and application programming interface (API), which is so-called Platform as a Service (PaaS). In this way, third party applications are also integrated into the architecture to form a high collaborative library information platform.

\subsection{Application Layer}

Application layer is directly user oriented. The platform provides various services to end users through this layer. The typical implementation is SOA [21], which is a loosely coupled architecture style. Other techniques included in this layer are SOAP [22], EJB [23], AJAX [24], and COBRA [25]. The applications include collaboration, unified communication and personal library.

\subsection{Access Interface Layer}

This layer provides a unified interface to access the system. The terminal connecting devices include multimedia classrooms, PC, laptops, smart phones, and tablets, etc. Based on the B/S structure, users only need a browser to to access all services [26]. 


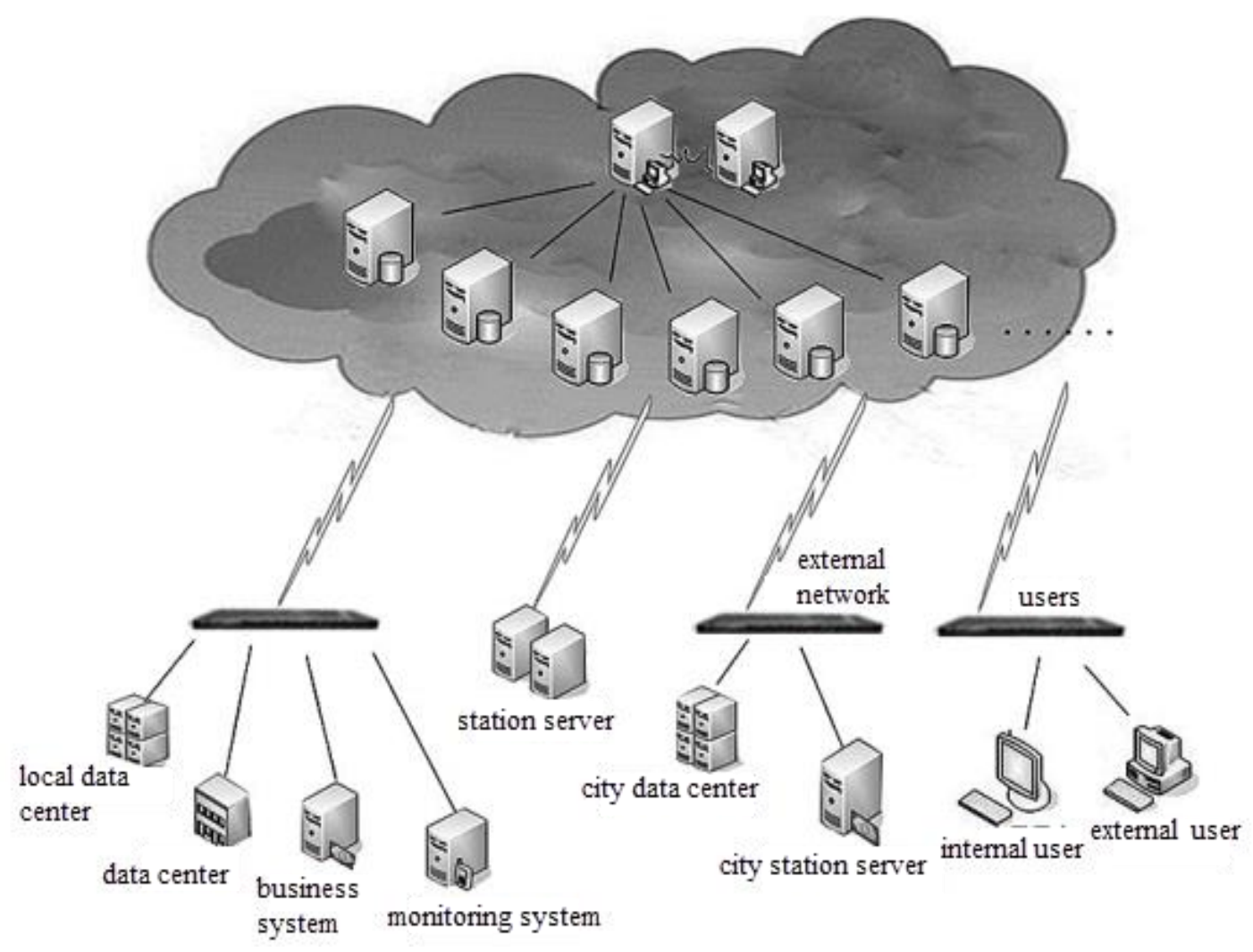

Figure 2. Physical Deployment of the Architecture

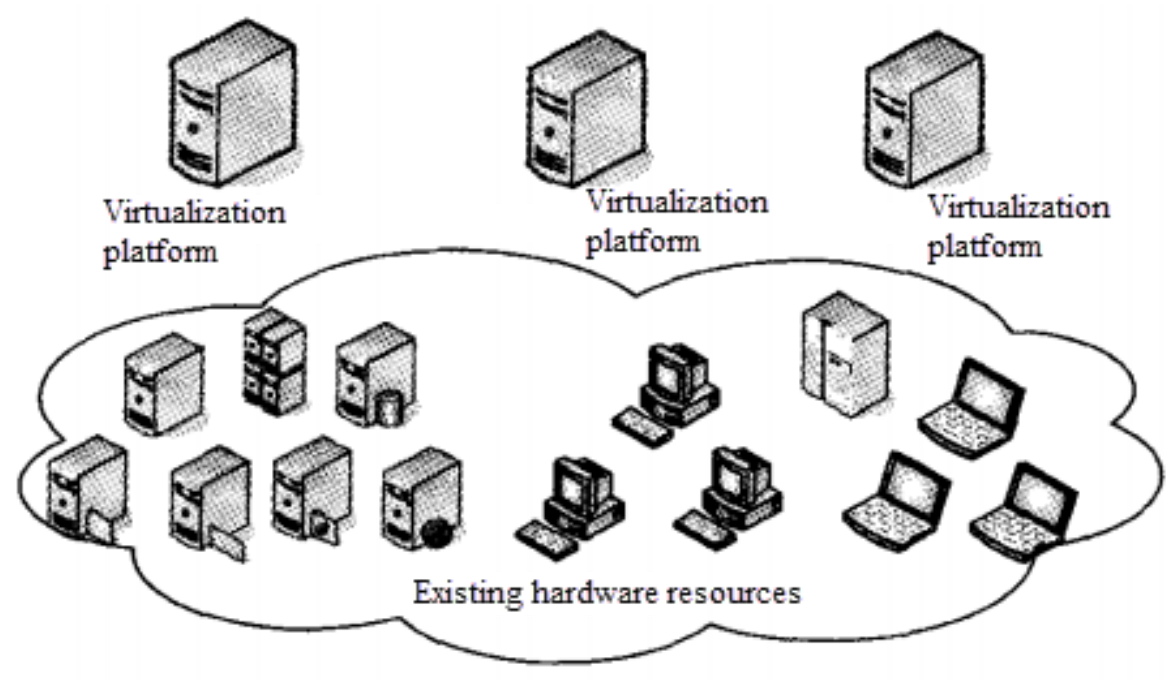

Figure 3. Illustration of Virtualization

\section{Resource Allocation}

In this section, we focus on one of the important issue in cloud based digital library architecture, that is, resource allocation.

We design a dynamic elastic resource allocation algorithm. The idea is to increase the CPU assignment when the utilization rate is high, and reclaim CPU resources when the utilization rate returns to the expected status. In this way, we can ensure maximum CPU resources are allocated and the corresponding utilization rate remains at a steady state. The algorithm is described in Algorithm 1. 


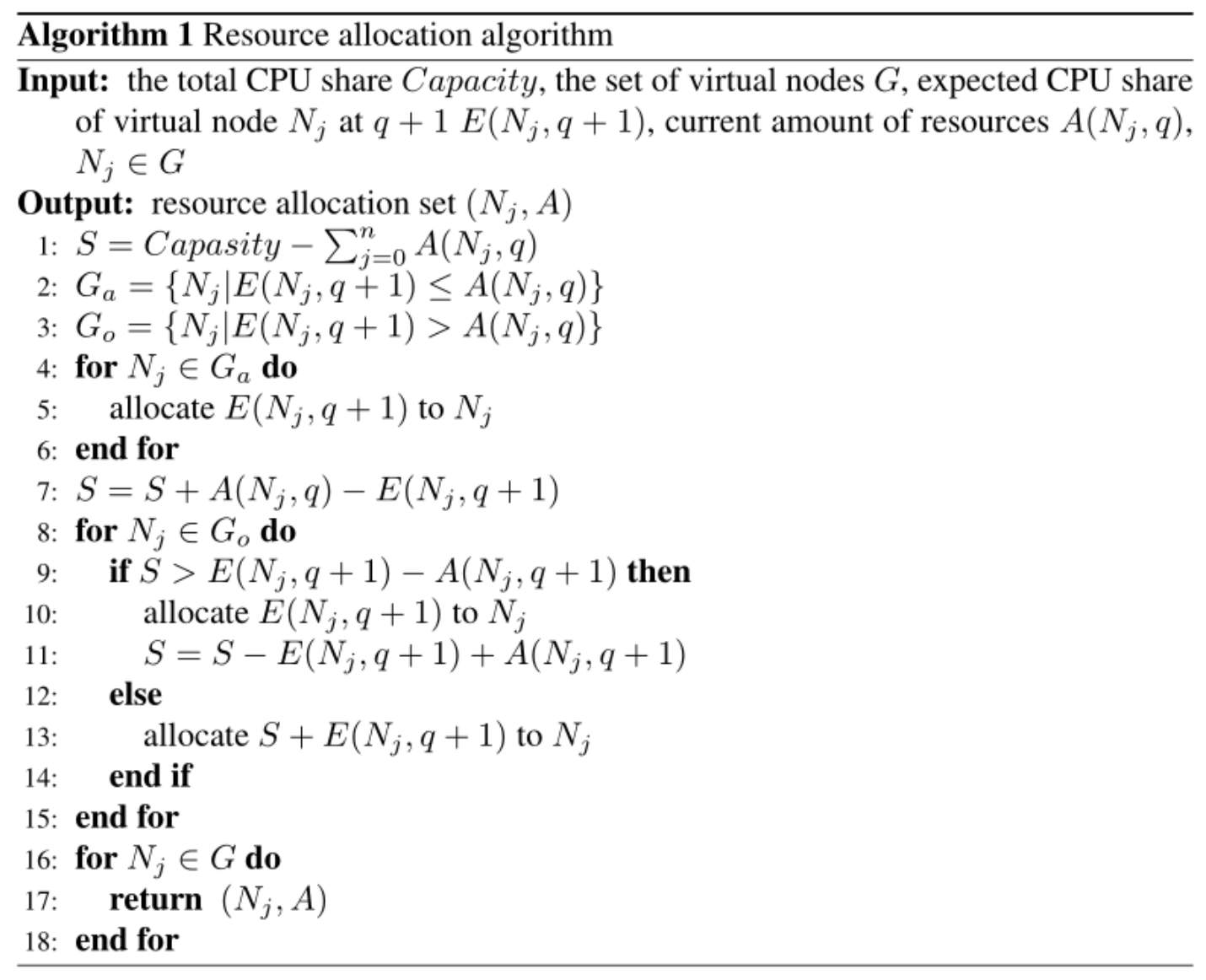

Figure 4. Algorithm Description of Resource Allocation

Suppose $E(q)$ be the expected CPU share assigned at time $q, A(q)$ be the actual CPU assigned at $q$, and $U(q)$ is the actual CPU used at $q \cdot r_{e}$ is the expected utilization rate of CPU, and $r(q)$ is the actual CPU utilization rate till $q$. Then the expected CPU assignment at $q-1$ can be calculated as follows:

$$
E(q+1)=A(q)-\frac{U(q)\left(r_{e}-r(q)\right)}{r_{e}} .
$$

In order to make sure the system stability, and avoid the frequent increasing or reducing resources assignment affected by some instantaneous peak, we introduce a satisfactory range of utilization rate. Let $r_{h}$ be the higher bound of utilization rate, and $r_{l}$ be the lower bound. Therefore, expected utilization rate $r_{e}$ subjects to $r_{l} \leq r_{e} \leq r_{h}$.

We add a controller to monitor the assignment of CPU resources. When the actual utilization rate is higher than $r_{h}$ or lower than $r_{l}$, the controller will look into the past $n$ records. If there exist $k$ events whose utilization rate is out of the satisfactory range, the controller will trigger re-assignment. In this way, we can easily avoid the instantaneous peaks.

\section{Experiment}

In this section, we evaluate our resource allocation algorithm. We use CloudSim [27] to simulate the cloud computing environment, and Intel Xeon CPU E5620 @ 2.40GHz as the virtualization hardware. 


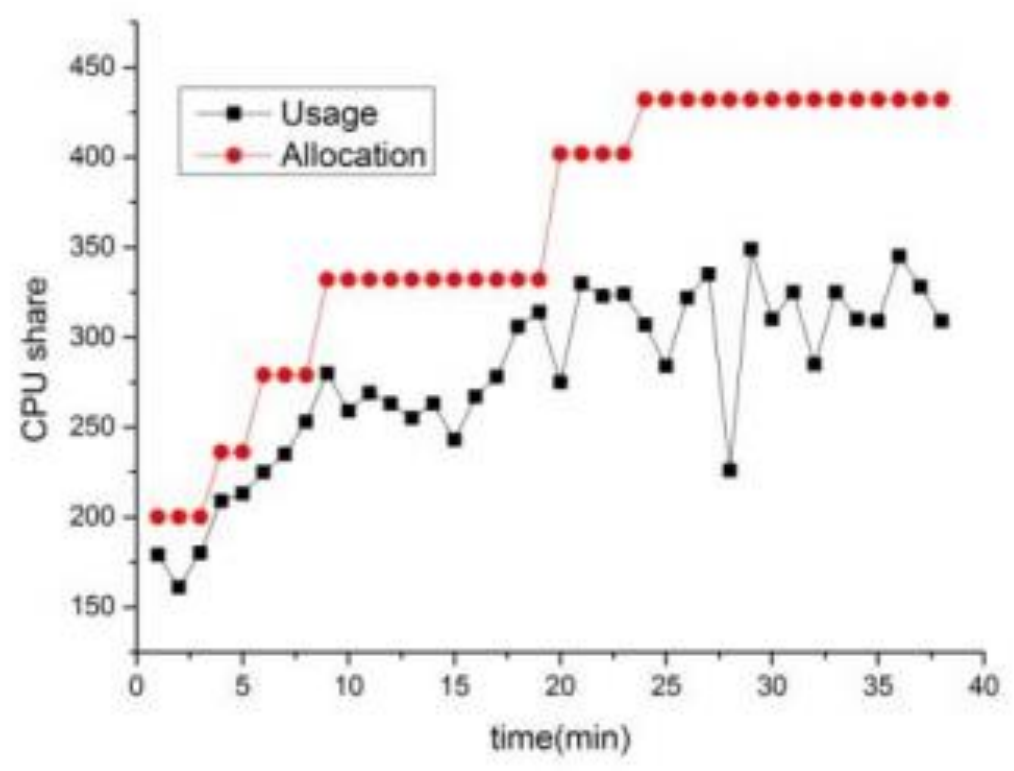

Figure 5. Resource Allocation when Workload Varies

We use the simple S4 benchmark framework in Yahoo S4 [28] as our testing framework. We simulated $4 \mathrm{M}$ events for test. The utilization rate settings of our algorithm are: $r_{e}=0.75, r_{h}=0.8, r_{l}=0.7$.

From Figure 5, we can observe that when the CPU usage is not stable, our allocation algorithm can adjust the CPU share to avoid trembles caused by instantaneous change of workload. For example, at the time of 20 minutes, the workload is smaller than the lower threshold. Therefore, the controller would look up the past $n$ usage of resources to adjust the allocation.

Figure 6 shows the actual utilization rate when running the application. We can see that generally the utilization is within the satisfactory range, which ensures the efficient allocation of resources.

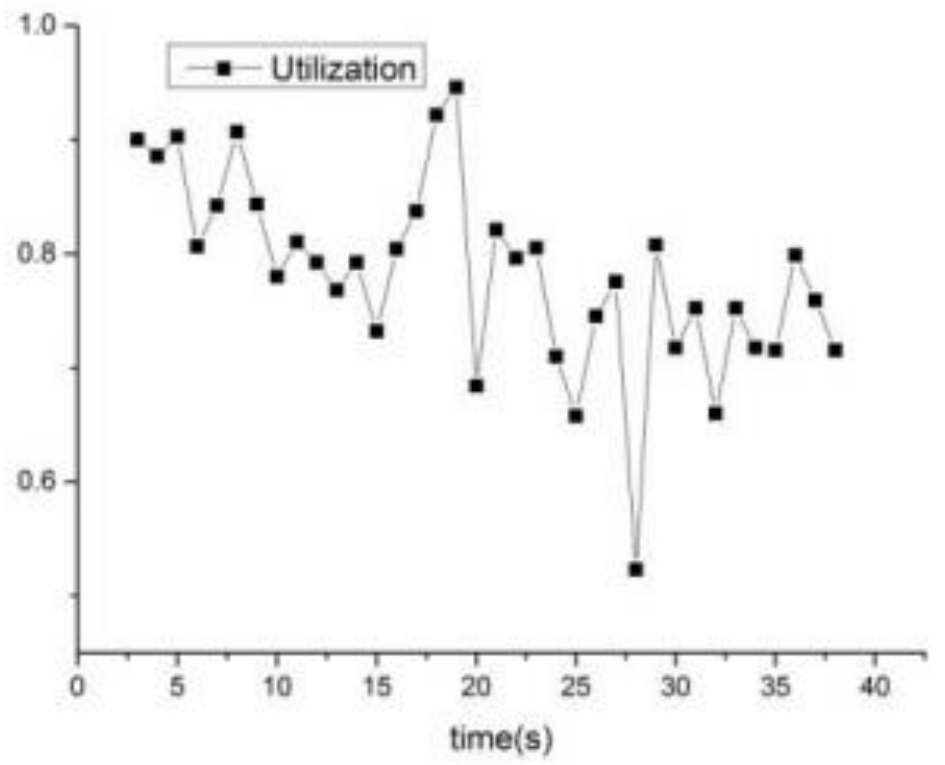

Figure 6. CPU Utilization Rate in Execution 


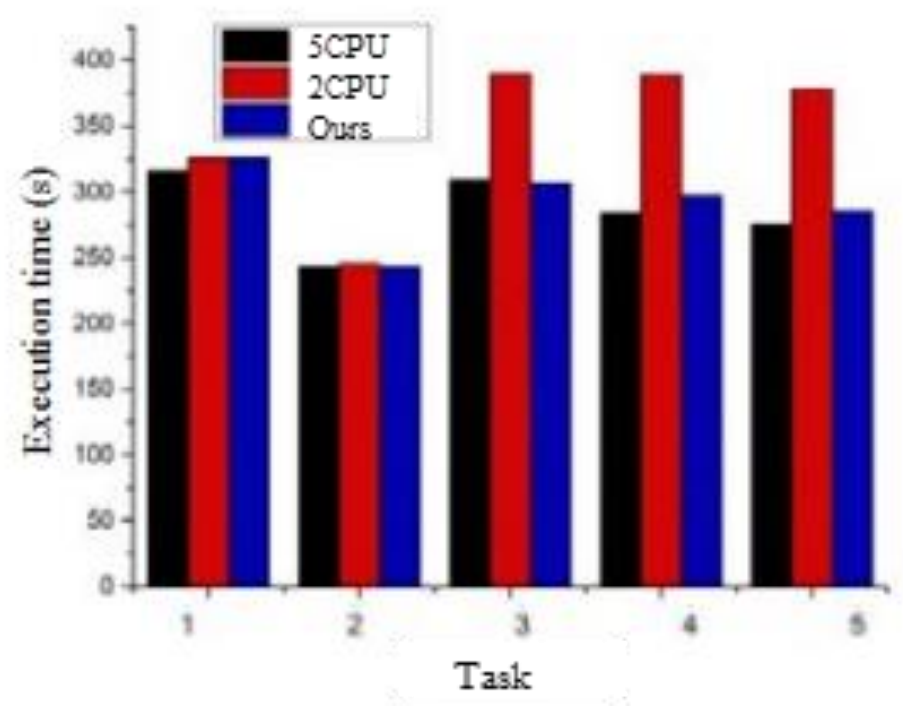

Figure 7. Performance of Different Allocation Strategies

Table 1. Description of Task Settings

\begin{tabular}{|c|c|c|}
\hline Task & Number of Events & Events per Second \\
\hline 1 & $2 \mathrm{M}$ & $8 \mathrm{~K}$ \\
\hline 2 & $2 \mathrm{M}$ & $12 \mathrm{~K}$ \\
\hline 3 & $4 \mathrm{M}$ & $20 \mathrm{~K}$ \\
\hline 4 & $4 \mathrm{M}$ & $22 \mathrm{~K}$ \\
\hline 5 & $4 \mathrm{M}$ & $24 \mathrm{~K}$ \\
\hline
\end{tabular}

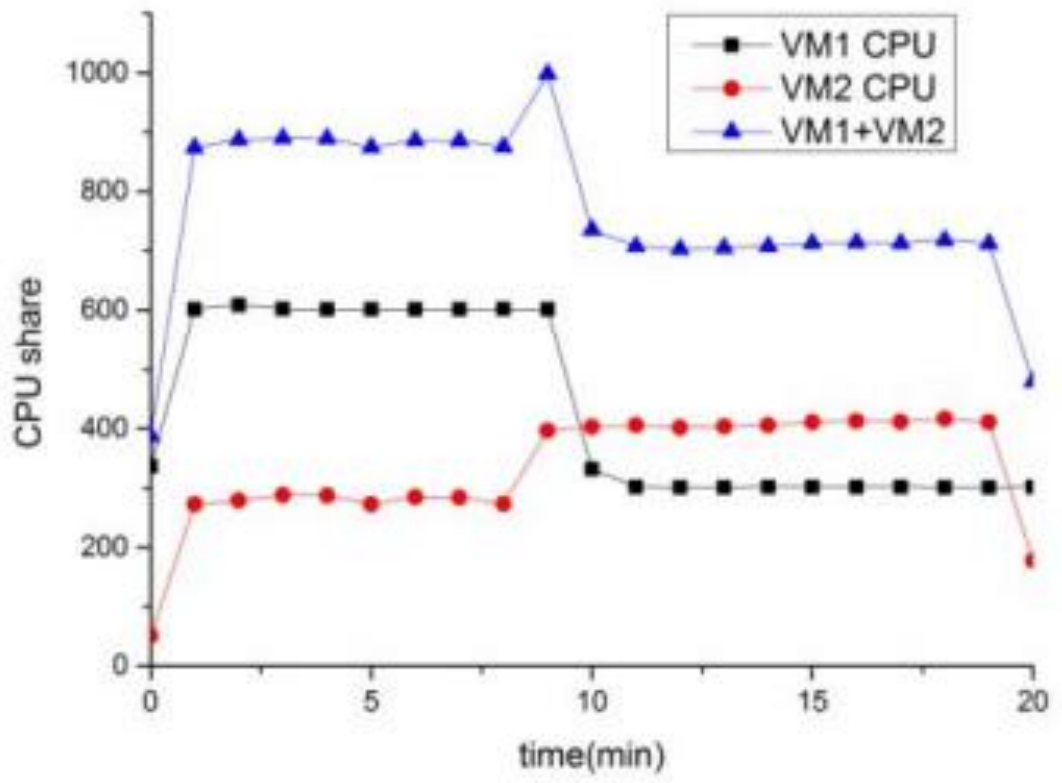

Figure 8. Performance of Different Allocation Strategies 


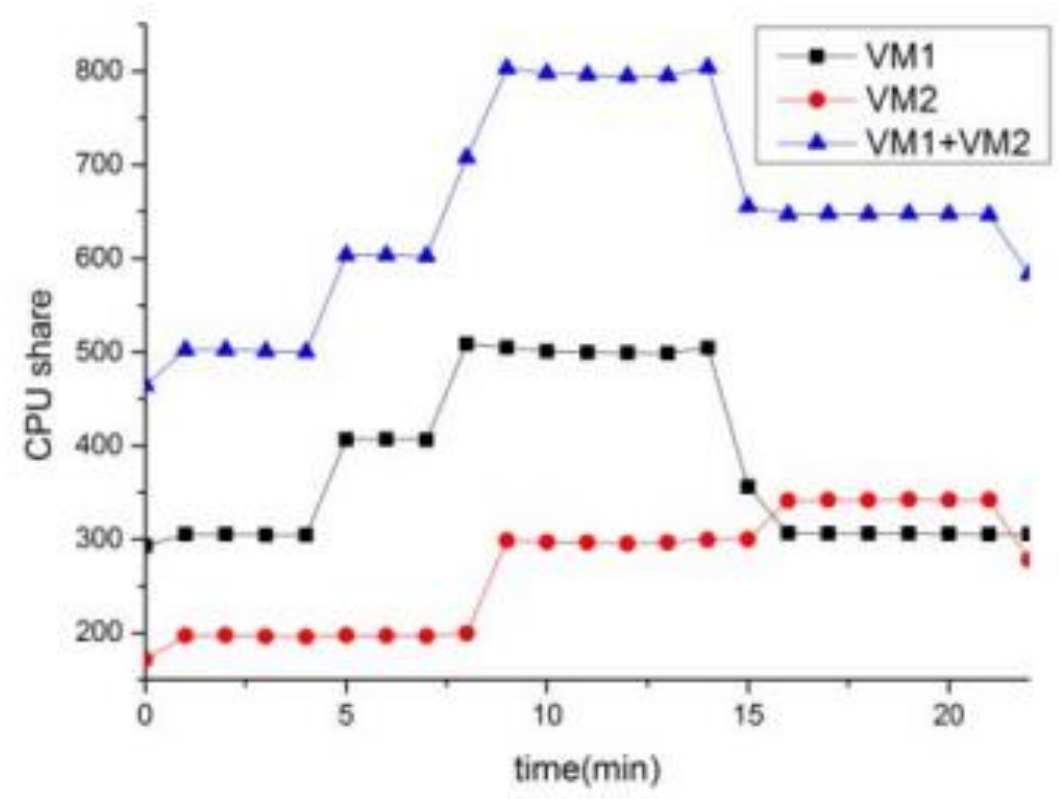

Figure 9. Performance of Different Allocation Strategies

We also compare the performance of our resource allocation strategy with the built-in method in Hadoop. We have five tasks as described in Table 1. The results are shown in Figure 7, where 5CPU denotes that 5 CPUs are available for allocation, and 2CPU denotes that only 2 CPUs can be used. We have the following observations. (1) For tasks 1 and 2, 2 CPUs are enough to meet the computing requirement, so the execution times for three strategies are almost the same. (2) For tasks 3, 4 and 5, the numbers of events are large. With typical allocation of Hadoop, 5CPU is much efficient than 2CPU. However, with our algorithm, we can still achieve good performance as the 5CPU.

Then we evaluate our algorithm on the single physical machine. Suppose we have two virtual machines on the same node, VM1 and VM2. Figure 8 gives the actual requirement of CPU share, and Figure 9 shows the allocation process. We can observe that, near the time 10 min, the algorithm can fully utilize all idle resources for computing tasks. This results means our algorithm is also efficient when allocating resources on a single node deployment.

\section{Conclusion}

Cloud computing brings chances for digital library construction. In this paper, we propose a reference architecture of a cloud based digital library platform. Specifically, we focus on the resource allocation based on virtualization technique, and design an elastic algorithm for resource allocation. We also conduct experiments to evaluate our algorithm. Results show that our algorithm exhibits good performance and high efficiency.

However, challenges still remain. For example, how to build customized personal libraries, and how to improve the security mechanism to avoid information disclosure, etc. Anyway, cloud computing is presenting new opportunities for constructing new library platform.

\section{Acknowledgements}

The authors would like to recognize the others who helped them and thank all our reviewers.

\section{References}

[1] M. Armbrust, "A view of cloud computing. Communications of the ACM, vol. 53, no. 4 (2010), pp. 50-58.

[2] P. Mell and T. Grance, "The NIST definition of cloud computing (draft)", NIST special publication, vol. 800, no. 145, (2011), pp. 7. 
[3] R. Buyya, "Cloud computing and emerging IT platforms: Vision, hype, and reality for delivering computing as the 5th utility", Future Generation computer systems, vol. 25, no.6, (2009), pp. 599-616.

[4] G. Lin, G. Dasmalchi and J. Zhu, "Cloud computing and IT as a service: opportunities and challenges", Web Services, 2008. ICWS'08. IEEE International Conference on. IEEE, (2008).

[5] M. Carroll, P. Kotzé and A. van der Merwe, "Securing Virtual and Cloud Environments", Cloud Computing and Services Science, Springer New York, (2012), pp. 73-90.

[6] P. Mell, and T. Grance, "The NIST definition of cloud computing (draft)", NIST special publication, vol. 800, no. 145, (2011), pp. 7.

[7] P. Buxmann, T. Hess and S. Lehmann, "Software as a Service", Wirtschaftsinformatik, vol. 50, no. 6, (2008), pp. 500-503.

[8] G. Lawton, "Developing software online with platform-as-a-service technology", Computer, vol. 41, no. 6, (2008), pp. 13-15.

[9] S. Bhardwaj, L. Jain and S. Jain, "Cloud computing: A study of infrastructure as a service (IAAS)", International Journal of engineering and information Technology, vol. 2, no. 1, (2010), pp. 60-63.

[10] J. Griffey, "Stranger Than We Know", Let Jason Griffey take you on a guided tour into the future of mobile computing, where access is ubiquitous and librarians ply their trade in the information cloud. NetConnect Library Journal. Online verfügbar unter http://www. libraryjournal. com/article/CA6599046. Html, (2008).

[11] M. Breeding, "Chapter 3: Major open source ILS products", Library technology reports, vol. 44, no. 8, (2008), pp. 16-31.

[12] M. Turner, D. Budgen and P. Brereton, "Turning software into a service”, Computer, vol. 36, no. 10, (2003), pp. 38-44.

[13] S. Shankar, "Amazon elastic compute cloud", (2009).

[14] E. Kroski, "Cloud Computing for Libraries", No. 11. ALA Store, (2012).

[15] M. R. Palankar, "Amazon S3 for science grids: a viable solution", Proceedings of the 2008 international workshop on Data-aware distributed computing, ACM, (2008).

[16] E. Mitchell, "Using cloud services for library IT infrastructure", Code4lib journal, vol. 9 (2010).

[17] A. Zahariev, “Google app engine”, Helsinki University of Technology, (2009).

[18] O. Bennett and K. Gilbert, "Extending liaison collaboration: partnering with faculty in support of a student learning community", Reference Services Review, vol. 37, no. 2, (2009), pp. 131-142.

[19] R. Fox, "Library in the clouds", OCLC Systems \& Services, vol. 25, no. 3, (2009), pp. 156-161.

[20] J. Jordan, "Climbing out of the box and into the cloud: Building web-scale for libraries", Journal of Library Administration, vol. 51, no. 1, (2010), pp. 3-17.

[21] E. Newcomer and G. Lomow, "Understanding SOA with web services (independent technology guides)", Addison-Wesley Professional, (2004).

[22] D. Box, "Simple object access protocol (SOAP), vol. 1, no. 1, (2000).

[23] E. Roman, R. P. Sriganesh and G. Brose, "Mastering enterprise javabeans", Wiley. com, (2005).

[24] J. J. Garrett, "Ajax: A new approach to web applications", (2005).

[25] X. He, "The SOA-Based Solution for Distributed Enterprise Application Integration. Computer Science-Technology and Applications", 2009. IFCSTA'09. International Forum on, vol. 3, (2009).

[26] T. O'reilly, "What is web", vol. 2, (2005).

[27] R. N. Calheiros, "CloudSim: a toolkit for modeling and simulation of cloud computing environments and evaluation of resource provisioning algorithms", Software: Practice and Experience, vol. 41, no. 1, (2011), pp. 23-50.

[28] L. Neumeyer, "S4: Distributed stream computing platform. Data Mining Workshops (ICDMW)", 2010 IEEE International Conference on. IEEE, (2010).

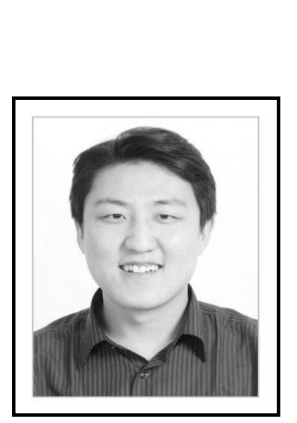

\section{Authors}

Yubin Wang, he received his bachelor's degree of engineering in Yanshan University, Qinhuangdao, Hebei. (2000) and master's degree of engineering in Yanshan University (2009), Now he is a lecturer in Hebei Normal University of Science \& Technology, Qinhuangdao, Hebei. His major fields of study are computer network technology. 


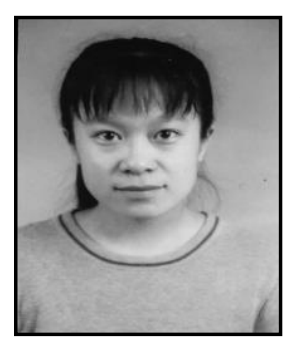

Jingyi Bo, she received her bachelor's degree of engineering in Hebei Normal University, Shijiazhuang, Hebei. (2002) and master's degree of engineering in Yanshan University (2008), Now she is a lecturer in Hebei Normal University of Science \& Technology, Qinhuangdao, Hebei. His major fields of study are computer network technology.

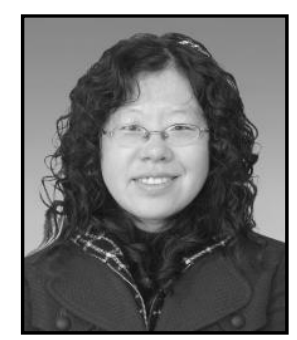

Weili Xu, her received her bachelor's degree of engineering in Hebei University, Tianjin. (2002) and master's degree of engineering in Yanshan University (2005), Now she is a lecturer in Hebei Normal University of Science \& Technology, Qinhuangdao, Hebei. His major fields of study are Information Retrieval. 
International Journal of Future Generation Communication and Networking Vol.7, No.3 (2014) 